\title{
Exact Solution of Thermoelastic Problem for a One-Dimensional Bar without Energy Dissipation
}

\author{
A. M. Abd El-Latief ${ }^{1}$ and S. E. Khader ${ }^{2}$ \\ ${ }^{1}$ Department of Mathematics, Faculty of Science, University of Alexandria, Egypt \\ ${ }^{2}$ Department of Mathematical and Theoretical Physics, Atomic Energy Authority, Egypt \\ Correspondence should be addressed to A. M. Abd El-Latief; m.abdellatief@yahoo.com
}

Received 3 November 2013; Accepted 26 November 2013; Published 4 February 2014

Academic Editors: J. K. Chen and X. H. Liu

Copyright (C) 2014 A. M. Abd El-Latief and S. E. Khader. This is an open access article distributed under the Creative Commons Attribution License, which permits unrestricted use, distribution, and reproduction in any medium, provided the original work is properly cited.

\begin{abstract}
We consider a homogeneous isotropic thermoelastic half-space in the context of the theory of thermoelasticity without energy dissipation. There are no body forces or heat source acting on the half-space. The surface of the half-space is affected by a time dependent thermal shock and is traction free. The Laplace transform with respect to time is used. The inverse transforms are obtained in an exact manner for the temperature, thermal stress, and displacement distributions. These solutions are represented graphically and discussed for several cases of the applied heating. Comparison is made between the predictions here and those of the theory of thermoelasticity with one relaxation time.
\end{abstract}

\section{Introduction}

In 1967, Lord and Shulman [1] introduced the theory of generalized thermoelasticity with one relaxation time for an isotropic body. This theory corrects the unrealistic conclusions of the older theories (the uncoupled and the coupled theories of thermoelasticity) that heat waves travel with infinite speeds. The Heat conduction law of this theory is the Cattaneo law which is different from Fourier's law utilized in both the coupled and the uncoupled theories. Among the contribution to the subject are the works in [26]. In 1972 Green and Lindsay [7] developed the theory of generalized thermoelasticity with two relaxation times, based on a generalized inequality of thermodynamics. In this theory both the equations of motion and of heat conduction are hyperbolic. The heat conduction law is the same as Fourier's law when the system has a centre of symmetry. Among the contributions to this theory are the works in $[8,9]$.

Green and Naghdi [10-12] have formulated three new models of thermoelasticity. In one of these models Green and Naghdi [12] predict that the internal rate of production of entropy is identically zero; that is, there is no dissipation of thermal energy. This theory (GN theory) is known as thermoelasticity without energy dissipation theory. In the development of this theory the thermal displacement gradient is considered as a constitutive variable, whereas in the conventional development of a thermoelasticity theory, the temperature gradient is taken as a constitutive variable [12]. A couple of uniqueness theorems have been proved in $[13,14]$, and one-dimensional waves in a half-space and in an unbounded body have been studied in $[15,16]$. In view of some experimental evidence available in favour of finiteness of heat propagation speed, generalized thermoelasticity theories are considered to be more realistic than the conventional theory in dealing with practical problems involving very large heat fluxes at short intervals, like those occurring in laser units and energy channels [17].

\section{Formulation of the Problem}

In this work, we consider a homogeneous isotropic and thermoelastic half-space in the context of the theory of thermoelasticity without energy dissipation. There are no body forces or heat source acting on the half-space taken as $x \geq 0$. The surface of the half-space is affected by a time dependent thermal shock and is traction free. 
From the physics of the problem, all functions will depend on the distance $x$ and the time $t$ only. The problem is thus onedimensional. The displacement components have the form

$$
\underline{\mathbf{u}}=(u(x, t), 0,0) .
$$

The basic equations due to Green and Nagdhi [12] in the absence of body forces and heat sources for isotropic elastic medium are given by the following:

(i) equation of motion:

$$
(\lambda+2 \mu) \frac{\partial^{2} u}{\partial x^{2}}-\gamma \frac{\partial T}{\partial x}=\rho \frac{\partial^{2} u}{\partial t^{2}},
$$

(ii) the equation of heat conduction:

$$
k^{*} \frac{\partial^{2} T}{\partial x^{2}}=\rho C_{E} \frac{\partial^{2} T}{\partial t^{2}}+\gamma T_{0} \frac{\partial^{3} u}{\partial t^{2} \partial x},
$$

(iii) the constitutive equation:

$$
\sigma_{x x}=\sigma=(\lambda+2 \mu) \frac{\partial u}{\partial x}-\gamma\left(T-T_{0}\right),
$$

where $\lambda$ and $\mu$ are Lamés modulii, $T$ is the absolute temperature of the medium, and $\gamma$ is a material constant given by $\gamma=(3 \lambda+2 \mu) \alpha_{t}$, where $\alpha_{t}$ is the coefficient of linear thermal expansion and $T_{0}$ is a reference temperature assumed to be such that $\left|\left(T-T_{0}\right) / T_{0}\right| \ll 1 . k^{*}$ is a material constant, characteristic of the theory and $C_{E}$ is the specific heat at constant strain.

Let us introduce the following nondimension variables:

$$
\begin{gathered}
x^{*}=c_{1} \eta x, \quad u^{*}=c_{1} \eta u, \quad t^{*}=c_{1}^{2} \eta t, \\
\sigma^{*}=\frac{\sigma}{\lambda+2 \mu}, \quad \theta=\frac{\gamma\left(T-T_{0}\right)}{(\lambda+2 \mu)} .
\end{gathered}
$$

The governing equations (2)-(4) in nondimensional form become (dropping the asterisks for convenience)

$$
\begin{gathered}
\frac{\partial^{2} u}{\partial x^{2}}-\frac{\partial \theta}{\partial x}=\frac{\partial^{2} u}{\partial t^{2}}, \\
C_{t}^{2} \frac{\partial^{2} \theta}{\partial x^{2}}=\frac{\partial^{2} \theta}{\partial t^{2}}+\varepsilon \frac{\partial^{3} u}{\partial t^{2} \partial x}, \\
\sigma=\frac{\partial u}{\partial x}-\theta,
\end{gathered}
$$

where $C_{t}^{2}=k^{*} / \rho c_{E} c_{1}^{2}$, and $\varepsilon=\gamma^{2} T_{0} / \rho c_{E}(\lambda+2 \mu)$.

\section{Solution of the Problem in the Laplace Transform Domain}

Applying the Laplace transform with parameter $s$ defined by the relation [14]

$$
\bar{f}(s)=\int_{0}^{\infty} f(t) e^{-s t} d t,
$$

to both sides of (6), we obtain

$$
\begin{gathered}
\frac{\partial^{2} \bar{u}}{\partial x^{2}}-\frac{\partial \bar{\theta}}{\partial x}=s^{2} \bar{u}, \\
C_{t}^{2} \frac{\partial^{2} \bar{\theta}}{\partial x^{2}}=s^{2} \bar{\theta}+\varepsilon s^{2} \frac{\partial \bar{u}}{\partial x}, \\
\bar{\sigma}=\frac{\partial \bar{u}}{\partial x}-\bar{\theta} .
\end{gathered}
$$

Eliminating $\bar{u}$ from (12) and (14), we get

$$
\left\{D^{4}-\frac{\left(C_{t}^{2}+1+\varepsilon\right)}{C_{t}^{2}} s^{2} D^{2}+\frac{s^{4}}{C_{t}^{2}}\right\} \bar{\theta}=0,
$$

where $D=\partial / \partial x$.

Equation (11) can be factorized as

$$
\left(D^{2}-k_{1}^{2}\right)\left(D^{2}-k_{2}^{2}\right) \bar{\theta}=0,
$$

where $k_{1}^{2}$ and $k_{2}^{2}$ are the roots of the characteristic equation

$$
k^{4}-\frac{\left(C_{t}^{2}+1+\varepsilon\right)}{C_{t}^{2}} s^{2} k^{2}+\frac{s^{4}}{C_{t}^{2}}=0 .
$$

$k_{1}^{2}$ and $k_{2}^{2}$ are given by

$$
k_{1}^{2}=s^{2}(B+A), \quad k_{2}^{2}=s^{2}(B-A),
$$

where $B=\left(C_{t}^{2}+1+\varepsilon\right) / 2 C_{t}^{2}$ and $A^{2}=\left(\left(C_{t}^{2}+1+\varepsilon\right) / 2 C_{t}^{2}\right)^{2}-$ $1 / C_{t}^{2}$.

The solutions of (12), bounded as $x$ tends to $\infty$ have the form

$$
\bar{\theta}=D_{1} e^{-k_{1} x}+D_{2} e^{-k_{2} x},
$$

where $D_{1}$ and $D_{2}$ are some parameters depending on $s$ only.

To calculate $\bar{u}$ using (9), we obtain

$$
\frac{\partial \bar{u}}{\partial x}=\frac{C_{t}^{2}}{\varepsilon s^{2}}\left[D_{1}\left(k_{1}^{2}-\frac{s^{2}}{C_{t}^{2}}\right) e^{-k_{1} x}+D_{2}\left(k_{2}^{2}-\frac{s^{2}}{C_{t}^{2}}\right) e^{-k_{2} x}\right] .
$$

Integrating both sides of (16) with respect to $x$, we get

$$
\bar{u}=\frac{-C_{t}^{2}}{\varepsilon s^{2}}\left[\frac{D_{1}}{k_{1}}\left(k_{1}^{2}-\frac{s^{2}}{C_{t}^{2}}\right) e^{-k_{1} x}+\frac{D_{2}}{k_{2}}\left(k_{2}^{2}-\frac{s^{2}}{C_{t}^{2}}\right) e^{-k_{2} x}\right] .
$$

Substituting (15) and (17) into (10), we obtain

$$
\begin{aligned}
\bar{\sigma}=\frac{C_{t}^{2}}{\varepsilon s^{2}}[ & D_{1}\left(k_{1}^{2}-\frac{s^{2}(1+\varepsilon)}{C_{t}^{2}}\right) e^{-k_{1} x} \\
& \left.+D_{2}\left(k_{2}^{2}-\frac{s^{2}(1+\varepsilon)}{C_{t}^{2}}\right) e^{-k_{2} x}\right] .
\end{aligned}
$$


The boundary conditions of the problem can be written as follows:

$$
\sigma=0, \quad \theta=F(t), \quad \text { at } x=0 .
$$

Applying the Laplace transform with parameter $s$ to both sides of (19), we get

$$
\bar{\theta}=\bar{F}(s), \quad \bar{\sigma}=0, \quad \text { at } x=0 .
$$

Substituting (15) and (18) into (20), we obtain the following system of linear equations in the unknown parameters $D_{1}$ and $D_{2}$ :

$$
\begin{gathered}
D_{1}+D_{2}=\bar{F}(s), \\
D_{1}\left(k_{1}^{2}-\frac{s^{2}(1+\varepsilon)}{C_{t}^{2}}\right)+D_{2}\left(k_{2}^{2}-\frac{s^{2}(1+\varepsilon)}{C_{t}^{2}}\right)=0 .
\end{gathered}
$$

The solution of the above equations is

$$
D_{1}=E \bar{F}(s), \quad D_{2}=(1-E) \bar{F}(s),
$$

where $E=\left(1+\varepsilon-C_{t}^{2}(B-A)\right) / 2 C_{t}^{2} A$.

Substituting (22) into (15), (17), and (18), we obtain

$$
\begin{gathered}
\bar{\theta}=\bar{F}(s)\left(E e^{-\sqrt{B+A} x s}+(1-E) e^{-\sqrt{B-A} x s}\right), \\
\bar{u}=\frac{-\bar{F}(s) C_{t}^{2}}{\varepsilon s}\left[\frac{E}{\sqrt{B+A}}\left(B+A-\frac{1}{C_{t}^{2}}\right) e^{-\sqrt{B+A} x s}\right. \\
\left.+\frac{1-E}{\sqrt{B-A}}\left(B-A-\frac{1}{C_{t}^{2}}\right) e^{-\sqrt{B-A} x s}\right], \\
\bar{\sigma}=\frac{\bar{F}(s) C_{t}^{2}}{\varepsilon}\left[E\left(B+A-\frac{1+\varepsilon}{C_{t}^{2}}\right) e^{-\sqrt{B+A} x s}\right. \\
\left.+(1-E)\left(B-A-\frac{1+\varepsilon}{C_{t}^{2}}\right) e^{-\sqrt{B-A} x s}\right] .
\end{gathered}
$$

The above equations can be written as

$$
\begin{gathered}
\bar{\theta}=\bar{F}(s)\left(E e^{-\mu_{1} s}+(1-E) e^{-\mu_{2} s}\right), \\
\bar{u}=\frac{-\bar{F}(s)}{s}\left[C_{1} e^{-\mu_{1} s}+C_{2} e^{-\mu_{2} s}\right], \\
\bar{\sigma}=\bar{F}(s)\left[C_{3} e^{-\mu_{1} s}+C_{4} e^{-\mu_{2} s}\right],
\end{gathered}
$$

where

$$
\begin{gathered}
C_{1}=\frac{C_{t}^{2}(1-E)}{\varepsilon \sqrt{B-A}}\left(B-A-\frac{1}{C_{t}^{2}}\right), \\
C_{2}=\frac{E C_{t}^{2}}{\varepsilon \sqrt{B+A}}\left(B+A-\frac{1}{C_{t}^{2}}\right), \\
C_{3}=\frac{C_{t}^{2} E\left(B+A-(1+\varepsilon) / C_{t}^{2}\right)}{\varepsilon}, \\
C_{4}=\frac{C_{t}^{2}(1-E)\left(B-A-(1+\varepsilon) / C_{t}^{2}\right)}{\varepsilon}, \\
\mu_{1}=\sqrt{B+A}, \quad \mu_{2}=\sqrt{B-A} .
\end{gathered}
$$

This completes the solution of the problem in the Laplace transform domain.

\section{Exact Solution in the Physical Domain}

We shall derive the temperature and stress distributions in an exact manner; by applying the inverse Laplace transform to both sides of (24) and (26), we get the following expressions:

$$
\begin{aligned}
\theta(x, t)= & E f\left(t-\mu_{1} x\right) H\left(t-\mu_{1} x\right) \\
& +(1-E) f\left(t-\mu_{2} x\right) H\left(t-\mu_{2} x\right), \\
\sigma(x, t)= & C_{3} f\left(t-\mu_{1} x\right) H\left(t-\mu_{1} x\right) \\
& +C_{4} f\left(t-\mu_{2} x\right) H\left(t-\mu_{2} x\right) .
\end{aligned}
$$

In obtaining the above equations, we have used the following property of the Laplace Transform:

$$
L^{-1}\left[\bar{F}(s) e^{-\alpha s}\right]=f(t-\alpha) H(t-\alpha),
$$

where $H(t-\mu)$ is the Heaviside unit step function; we have

$$
\begin{aligned}
L^{-1}\left[\frac{\bar{F}(s) e^{-\alpha s}}{s}\right] & =\int_{0}^{t}\left[L^{-1} \bar{F}(s) e^{-\alpha s}\right]_{t=\tau} d \tau \\
& =\int_{0}^{t} f(\tau-\alpha) H(\tau-\alpha) d \tau .
\end{aligned}
$$

According to the definition of Heaviside unit step function, we have two cases:

(i) if $\mu<t$, then the integral right hand side of (31) becomes $\int_{\mu}^{t} f(\tau-\mu) d \tau$;

(ii) while if $\mu>t$ in this case the integral vanishes; thus (31) becomes

$$
L^{-1}\left[\frac{\bar{F}(s) e^{-\alpha s}}{s}\right]=H(\tau-\alpha) \int_{\mu}^{t} f(\tau-\alpha) d \tau .
$$

Now, using (32) and (25), we obtain the exact form of the displacement distribution in the form

$$
\begin{aligned}
u(x, t)=-[ & C_{1} H\left(t-\mu_{1} x\right) \int_{\mu_{1} x}^{t} f\left(\tau-\mu_{1} x\right) d \tau \\
& \left.+C_{2} H\left(t-\mu_{2} x\right) \int_{\mu_{2} x}^{t} f\left(\tau-\mu_{2} x\right) d \tau\right] .
\end{aligned}
$$

This completes the solution of the problem in the physical domain.

\section{Numerical Results and Discussion}

We will consider the solution of the problem for different choices for the function $f(t)$ as shown in Table 1 , where $\theta_{0}$ and $a$ are constants.

Substituting $f(t)$ into (29), (30), and (33), we obtain the following. 
TABle 1

\begin{tabular}{lc}
\hline Case & $f(t)$ \\
\hline 1 & $\theta_{0} H(t)$ \\
2 & $e^{-t}$ \\
3 & $H(t-a) \sin \left(\frac{\pi t}{a}\right)$ \\
\hline
\end{tabular}

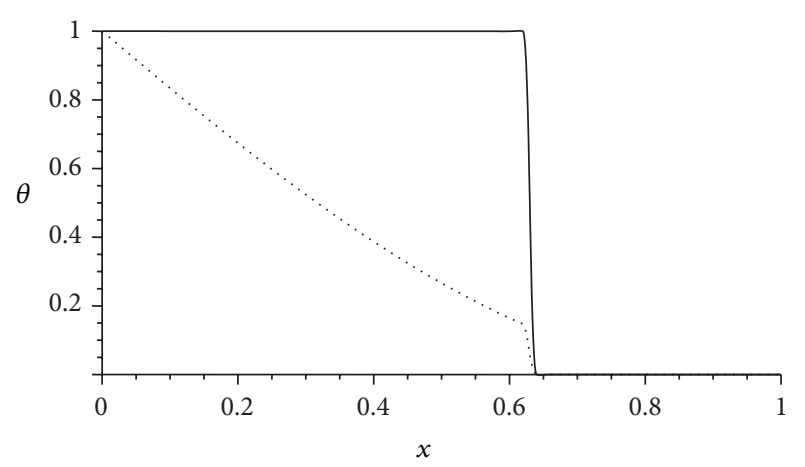

..... LS theory

— GN theory

FIgURE 1: Temperature distribution for Case 1.

Case 1. Consider the following:

$$
\begin{array}{r}
\theta(x, t)=E H\left(t-\mu_{1} x\right)+(1-E) H\left(t-\mu_{2} x\right) \\
\sigma(x, t)=C_{3} H\left(t-\mu_{1} x\right)+C_{4} H\left(t-\mu_{2} x\right) \\
u(x, t)=-\left[C_{1}\left(t-\mu_{1} x\right) H\left(\tau-\mu_{1} x\right)\right. \\
\left.+C_{2}\left(t-\mu_{2} x\right) H\left(\tau-\mu_{2} x\right)\right] .
\end{array}
$$

Case 2. Consider the following:

$$
\begin{aligned}
\theta(x, t)= & E e^{-\left(t-\mu_{1} x\right)} H\left(t-\mu_{1} x\right) \\
& +(1-E) e^{-\left(t-\mu_{2} x\right)} H\left(t-\mu_{2} x\right), \\
\sigma(x, t)= & C_{3} e^{-\left(t-\mu_{1} x\right)} H\left(t-\mu_{1} x\right) \\
& +C_{4} e^{-\left(t-\mu_{2} x\right)} H\left(t-\mu_{2} x\right), \\
u(x, t)= & -C_{1} H\left(t-\mu_{1} x\right)\left\lfloor 1-e^{-\left(t-\mu_{1} x\right)}\right\rfloor \\
& -C_{2} H\left(t-\mu_{2} x\right)\left\lfloor 1-e^{-\left(t-\mu_{2} x\right)}\right\rfloor .
\end{aligned}
$$

Case 3. Consider the following:

$$
\begin{aligned}
\theta= & E H\left(t-\mu_{1} x\right) H(a-t) \sin \left(\frac{\pi\left(t-\mu_{1} x\right)}{a}\right) \\
& +(1-E) H\left(t-\mu_{2} x\right) H(a-t) \sin \left(\frac{\pi\left(t-\mu_{2} x\right)}{a}\right), \\
\sigma= & C_{3} H\left(t-\mu_{1} x\right) H(a-t) \sin \left(\frac{\pi\left(t-\mu_{1} x\right)}{a}\right) \\
& +C_{4} H\left(t-\mu_{2} x\right) H(a-t) \sin \left(\frac{\pi\left(t-\mu_{2} x\right)}{a}\right),
\end{aligned}
$$

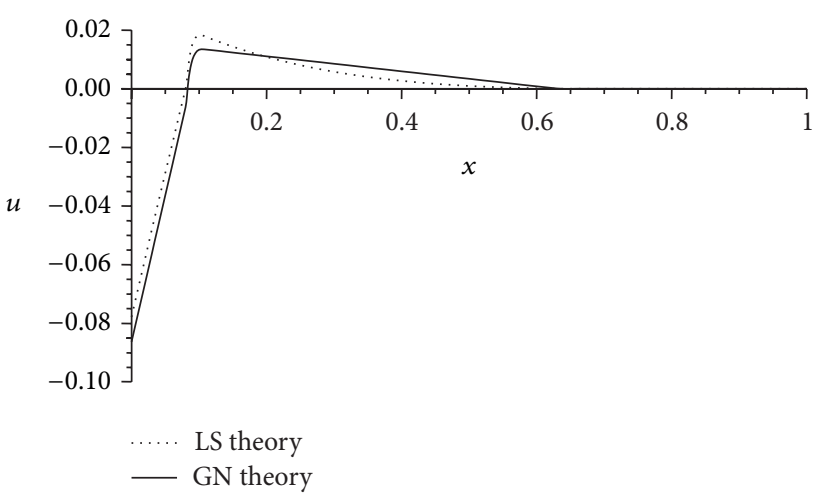

Figure 2: Displacement distribution for Case 1.

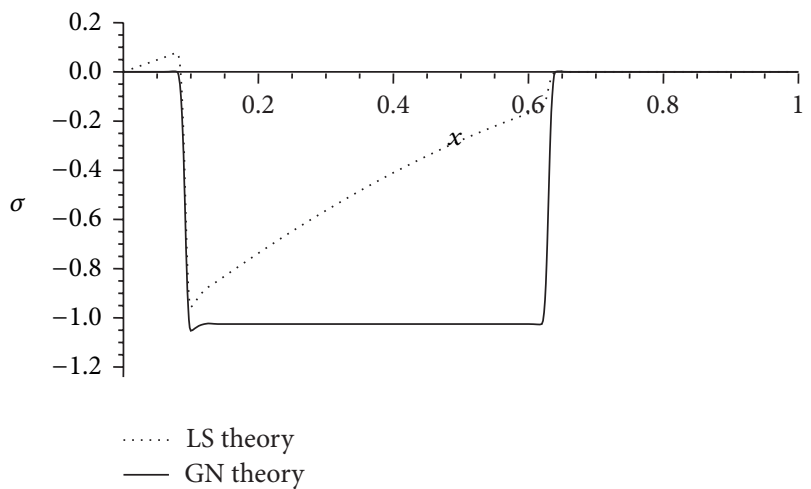

FIgURE 3: Stress distribution for Case 1.

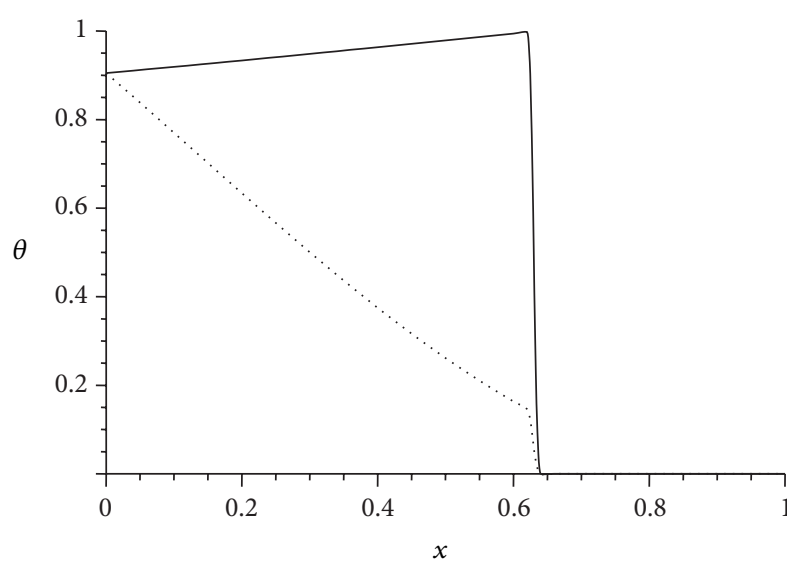

LS theory

GN theory

FIgURE 4: Temperature distribution for Case 2.

$$
\begin{aligned}
u=\frac{a}{\pi}( & C_{1} H\left(t-\mu_{1} x\right) H(a-t) \\
& \times\left[\cos \left(\frac{\pi\left(t-\mu_{1} x\right)}{a}\right)-1\right] \\
& +C_{2} H\left(t-\mu_{2} x\right) H(a-t) \\
& \left.\times\left[\cos \left(\frac{\pi\left(t-\mu_{2} x\right)}{a}\right)-1\right]\right) .
\end{aligned}
$$




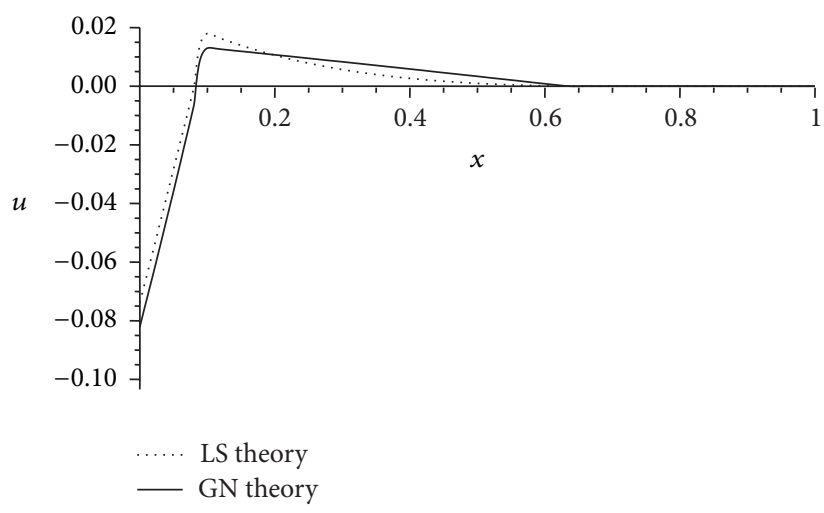

Figure 5: Displacement distribution for Case 2.

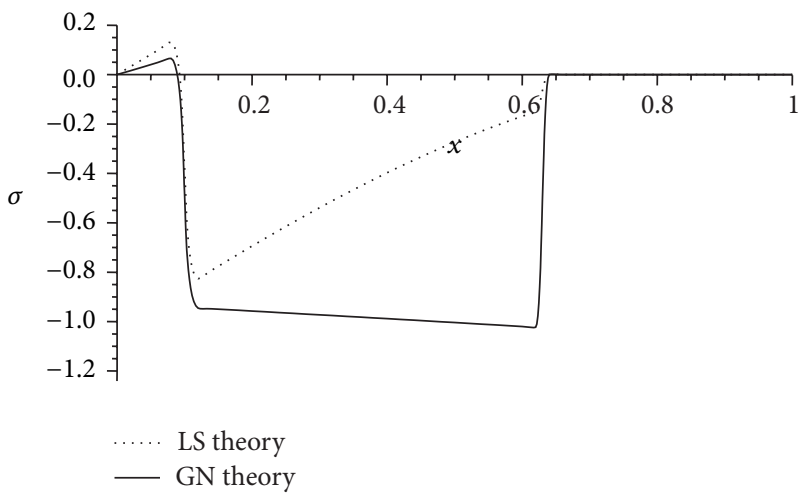

Figure 6: Stress distribution for Case 2.

Obviously the above exact solution verifies the boundary conditions introduced in (19) for all different cases.

We shall now compare, graphically, our results, where the theory of thermoelasticity without energy dissipation (GN theory) was used with the results of the solution of a similar problem in [18], where the generalized theory of thermoelasticity due to Lord-Shulman (LS theory) was used. Due to the nature of the characteristic roots of the LS theory, the authors of [18] could not obtain a closed form exact solution. They used asymptotic expansions valid for small values of time.

For purposes of numerical evaluations, the calculations were performed for a copper-like material for which [19]

$$
\varepsilon=0.0168, \quad C_{t}^{2}=40, \quad \theta_{0}=1, \quad a=0.04 .
$$

The temperature, displacement, and stress distributions are plotted for one value of time, namely, for $t=0.1$. In all figures, LS theory is represented by dotted lines, while GN theory is represented by solid lines.

For the three cases, the temperature distributions are shown in Figures 1, 4, and 7, the displacement distributions are shown in Figures 2, 5, and 8, while the stress distributions are shown in Figures 3, 6, and 9.

At the locations $x_{1}=t / \mu_{1}$ and $x_{2}=t / \mu_{2}$ the temperature and stress distributions have two finite jumps, while the displacement is continuous everywhere having a discontinuous first derivative at the above locations. The first

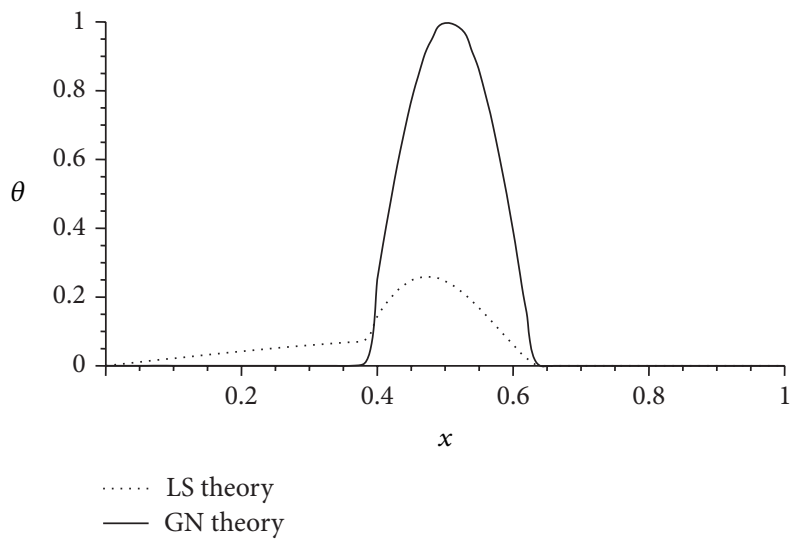

FIgURE 7: Temperature distribution for Case 3.

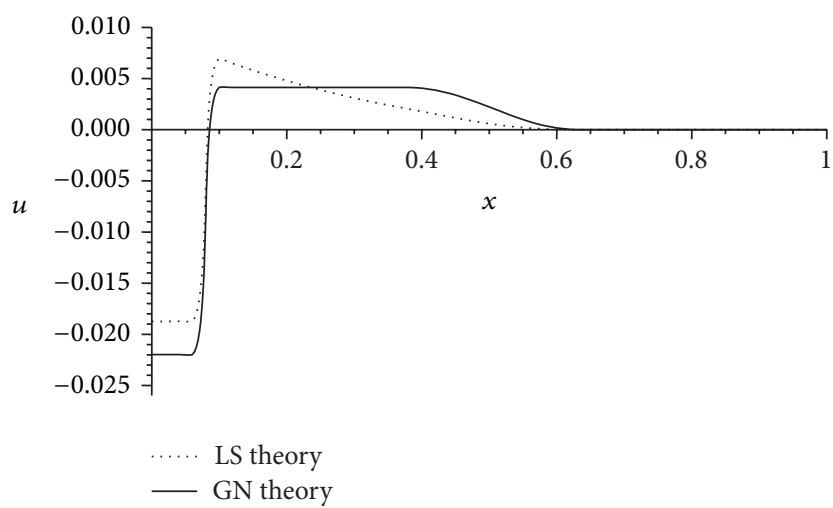

Figure 8: Displacement distribution for Case 3.

discontinuity is not shown in Figures 1, 4, and 7 because it is very small $\left(E \approx 4(10)^{-4}\right)$.

$x_{1}$ and $x_{2}$ are the locations of the wave fronts. We thus have two waves propagating into the medium from the surface of the half space. The first is mainly mechanical and has velocity equal to $v_{1}=1 / \mu_{1}$. The second is mainly thermal and has velocity equal to $v_{2}=1 / \mu_{2}$.

For $x>x_{2}$ the solution for all functions is identically zero. Thus, the solution is confined to a finite region of space and does not extend to infinity. This is not the case for the coupled and the uncoupled theories of thermoelasticity, where the solution extends to infinity instantaneously signifying an infinite speed of propagation of waves. The values of the velocities and locations of the wave fronts are as follows:

$$
\begin{gathered}
v_{1}=0.999784687321833, \\
v_{2}=6.32591737054768, \\
x_{1}=0.09997846873218333, \\
x_{2}=0.632591737054768 .
\end{gathered}
$$

The difference between the predictions of the theories of LS and GN is most apparent in the graphs of the temperature distribution. In the LS theory the temperature 


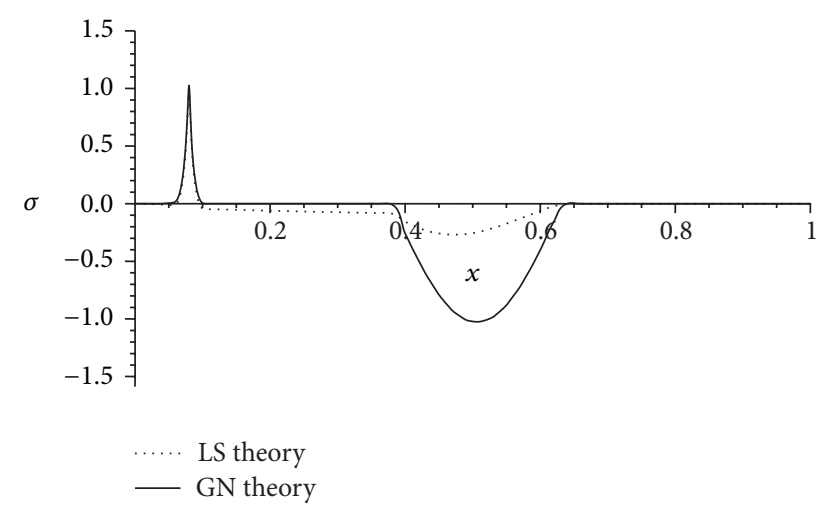

FIgURE 9: Stress distribution for Case 3.

decreases monotonically signifying continuous dissipation of heat energy. This is not the case for GN theory.

\section{Conflict of Interests}

The authors declare that there is no conflict of interests regarding the publication of this paper.

\section{References}

[1] H. Lord and Y. Shulman, "A generalized dynamical theory of thermo-elasticity," Journal of the Mechanics and Physics of Solids, vol. 15, no. 5, pp. 299-309, 1967.

[2] H. Sherief and N. M. El-Maghraby, "An internal penny-shaped crack in an infinite thermoelastic solid," Journal of Thermal Stresses, vol. 26, no. 4, pp. 333-352, 2003.

[3] H. Sherief and F. A. Hamza, "Generalized two-dimensional thermoelastic problems in spherical regions under axisymmetric distributions," Journal of Thermal Stresses, vol. 19, no. 1, pp. 55-76, 1996.

[4] H. Sherief and M. Anwar, "A problem in generalized thermoelasticity for an infinitely long annular cylinder composed of two different materials," Acta Mechanica, vol. 80, no. 1-2, pp. 137-149, 1989.

[5] M. Anwar and H. Sherief, "Boundary integral equation formulation of generalized thermoelasticity in a Laplace-transform domain," Applied Mathematical Modelling, vol. 12, no. 2, pp. 161$166,1988$.

[6] H. Sherief and M. A. Ezzat, "Solution of the generalized problem of thermoelasticity in the form of series of functions," Journal of Thermal Stresses, vol. 17, no. 1, pp. 75-95, 1994.

[7] A. E. Green and K. A. Lindsay, "Thermoelasticity," Journal of Elasticity, vol. 2, no. 1, pp. 1-7, 1972.

[8] H. Sherief, "State space approach to thermoelasticity with two relaxation times," International Journal of Engineering Science, vol. 31, no. 8, pp. 1177-1189, 1993.

[9] H. Sherief, "Problem in electromagneto thermoelasticity for an infinitely long solid conducting circular cylinder with thermal relaxation," International Journal of Engineering Science, vol. 32, no. 7, pp. 1137-1149, 1994.

[10] A. E. Green and P. M. Naghdi, "A re-examination of the basic postulate of thermomechanics," Proceedings of the Royal Society A, vol. 432, no. 1885, pp. 171-194, 1991.
[11] A. E. Green and P. M. Naghdi, "On undamped heat waves in an elastic solid," Journal of Thermal Stresses, vol. 15, no. 2, pp. 253-264, 1992.

[12] A. E. Green and P. M. Naghdi, “Thermoelasticity without energy dissipation," Journal of Elasticity, vol. 31, no. 3, pp. 189-208, 1993.

[13] D. S. Chandrasekharaiah, "A uniqueness theorem in the theory of thermoelasticity without energy dissipation," Journal of Thermal Stresses, vol. 19, no. 3, pp. 267-272, 1996.

[14] D. S. Chandrasekharaiah, "A note on the uniqueness of solution in the linear theory of thermoelasticity without energy dissipation," Journal of Elasticity, vol. 43, no. 3, pp. 279-283, 1996.

[15] D. S. Chandrasekharaiah, "One-dimensional wave propagation in the linear theory of thermoelasticity without energy dissipation," Journal of Thermal Stresses, vol. 19, no. 8, pp. 695-710, 1996.

[16] D. S. Chandrasekharaiah, "Thermoelastic plane waves without energy dissipation," Mechanics Research Communications, vol. 23, no. 5, pp. 549-555, 1996.

[17] D. S. Chandrasekharaiah, "Thermoelasticity with second sound: a review," Applied Mechanics Reviews, vol. 39, no. 3, pp. 355-376, 1986.

[18] R. Dhaliwal and H. Sherief, "A reciprocity theorem and integral representation for generalized thermoelasticity," Journal of Mathematical and Physical Sciences, vol. 15, pp. 537-552, 1981.

[19] H. Sherief and H. Saleh, "A problem for an infinite thermoelastic body with a spherical cavity," International Journal of Engineering Science, vol. 36, no. 4, pp. 473-487, 1998. 

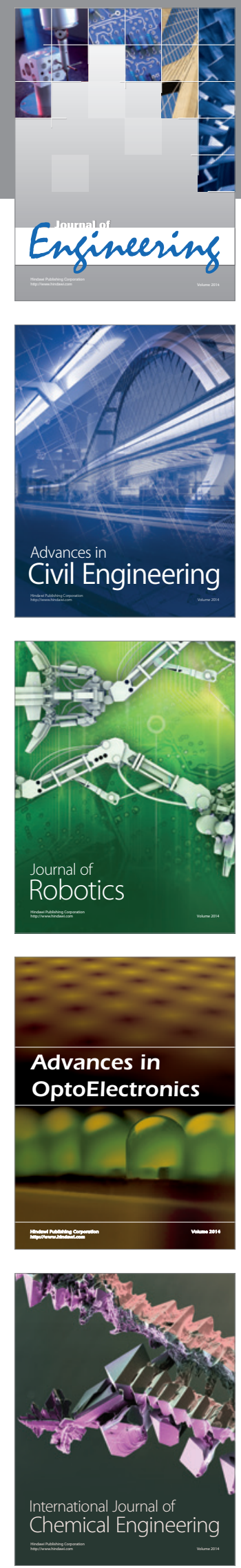

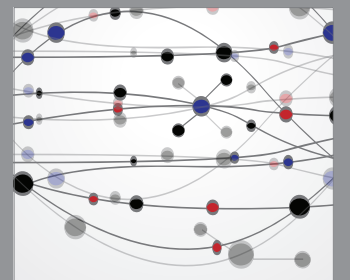

The Scientific World Journal
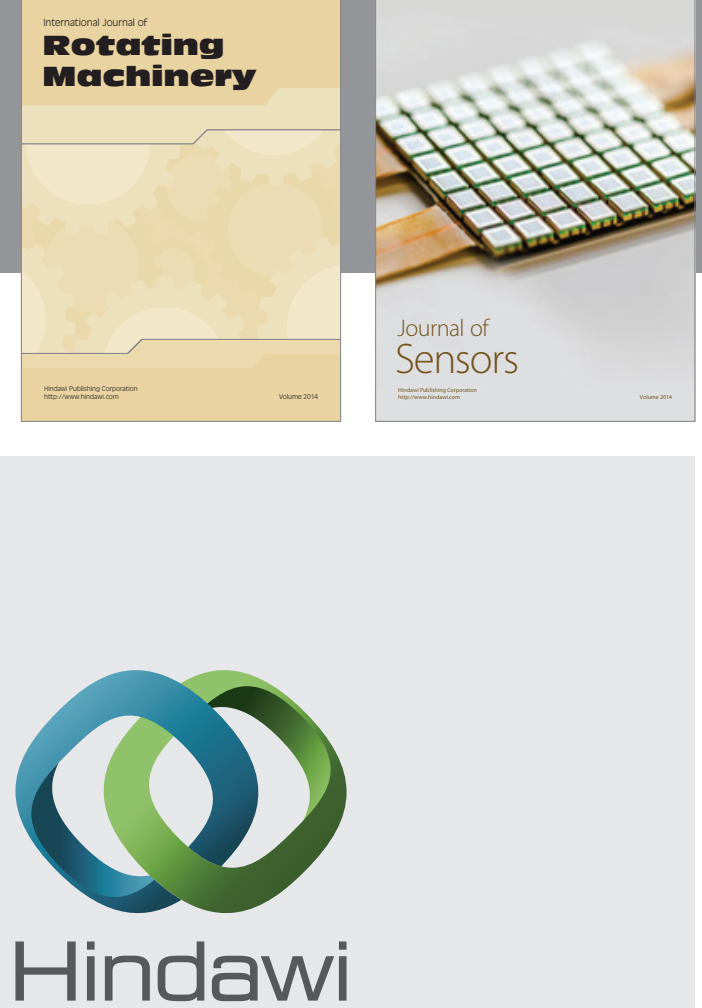

Submit your manuscripts at http://www.hindawi.com
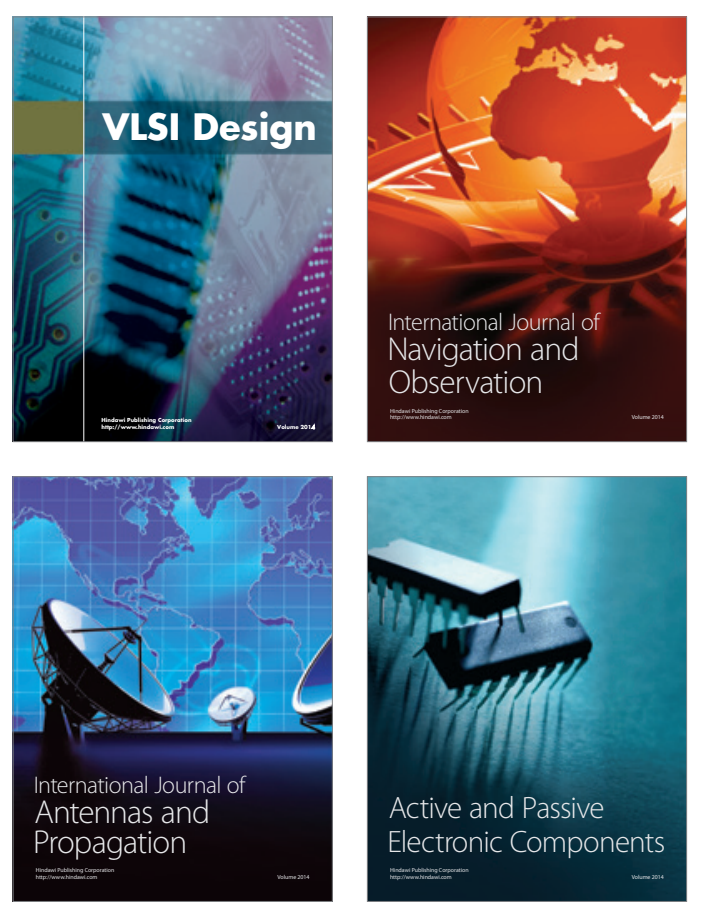
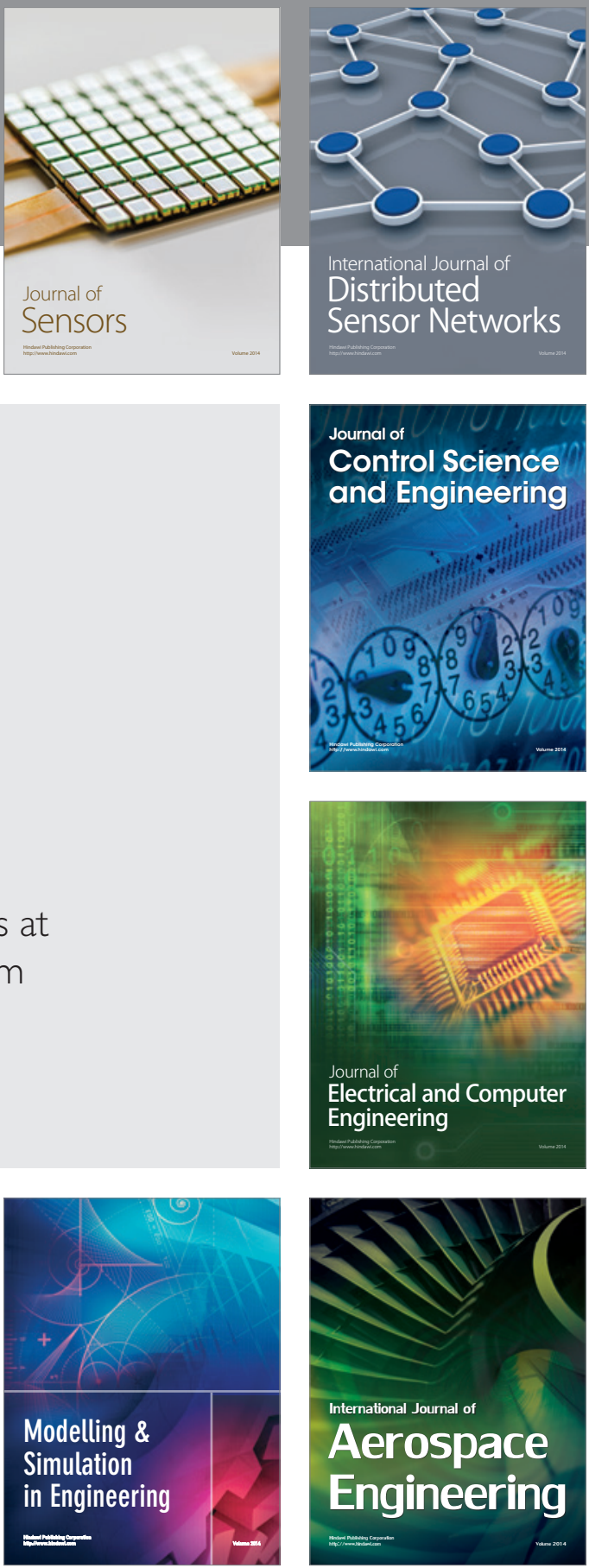

Journal of

Control Science

and Engineering
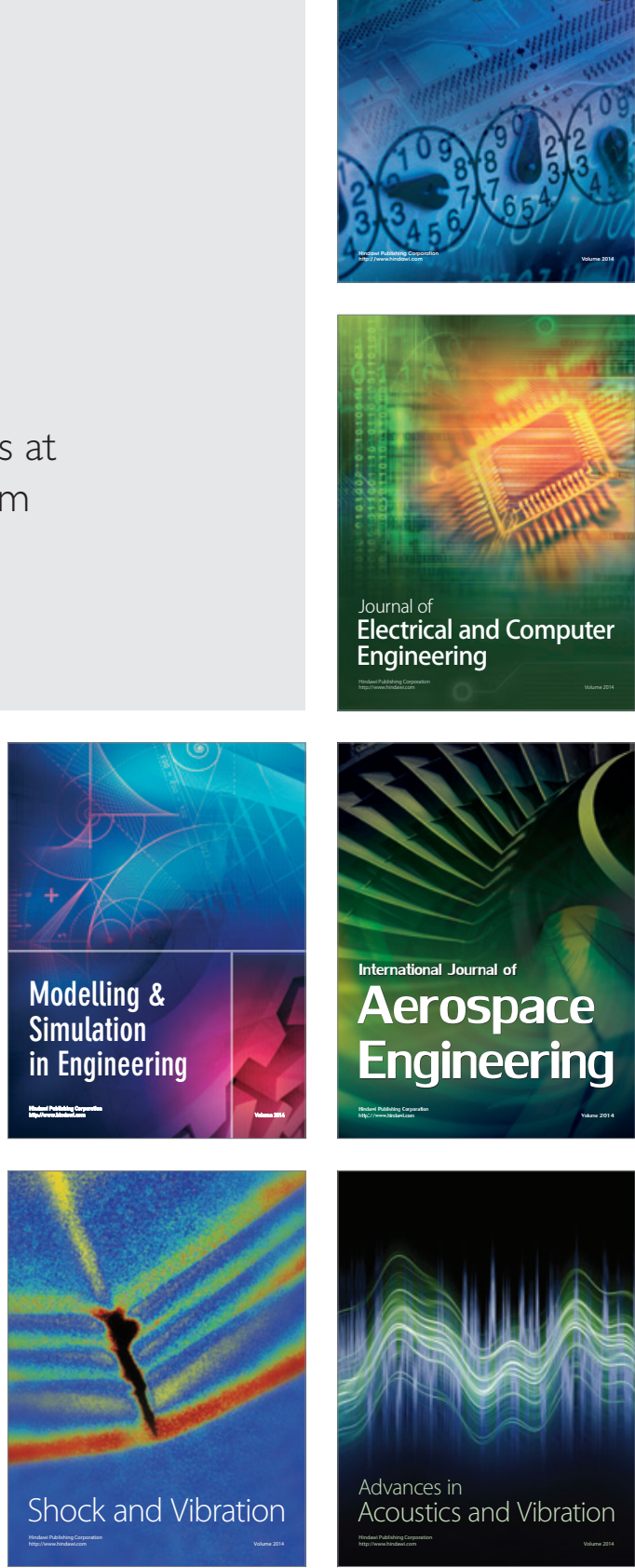